\title{
The background Friedmannian Hubble constant in relativistic inhomogeneous cosmology and the age of the Universe
}

\author{
Boudewijn F. Roukema ${ }^{1,2}$, Pierre Mourier ${ }^{2,3}$, Thomas Buchert ${ }^{2}$, and Jan J. Ostrowski ${ }^{1,2}$ \\ 1 Toruń Centre for Astronomy, Faculty of Physics, Astronomy and Informatics, Grudziadzka 5, Nicolaus Copernicus University, \\ ul. Gagarina 11, 87-100 Toruń, Poland \\ e-mail: boud@astro.uni.torun.pl \\ 2 Univ. Lyon, Ens de Lyon, Univ. Lyon1, CNRS, Centre de Recherche Astrophysique de Lyon UMR 5574, 69007 Lyon, France \\ 3 Département de Physique, École normale supérieure, 24 rue Lhomond, 75230 Paris Cedex 05, France
}

Received 9 October 2016 / Accepted 27 November 2016

\begin{abstract}
Context. In relativistic inhomogeneous cosmology, structure formation couples to average cosmological expansion. A conservative approach to modelling this assumes an Einstein-de Sitter model (EdS) at early times and extrapolates this forward in cosmological time as a "background model" against which average properties of today's Universe can be measured.

Aims. This modelling requires adopting an early-epoch-normalised background Hubble constant $H_{1}^{\text {bg }}$.

Methods. Here, we show that the $\Lambda$ CDM model can be used as an observational proxy to estimate $H_{1}^{\text {bg }}$ rather than choose it arbitrarily. We assume (i) an EdS model at early times; (ii) a zero dark energy parameter; (iii) bi-domain scalar averaging-division of the spatial sections into over- and underdense regions; and (iv) virialisation (stable clustering) of collapsed regions.

Results. We find $H_{1}^{\mathrm{bg}}=37.7 \pm 0.4 \mathrm{~km} \mathrm{~s}^{-1} / \mathrm{Mpc}$ (random error only) based on a Planck $\Lambda$ CDM observational proxy.

Conclusions. Moreover, since the scalar-averaged expansion rate is expected to exceed the (extrapolated) background expansion rate, the expected age of the Universe should be much younger than $2 /\left(3 H_{1}^{\mathrm{bg}}\right)=17.3 \mathrm{Gyr}$. The maximum stellar age of Galactic bulge microlensed low-mass stars (most likely: $14.7 \mathrm{Gyr}$; $68 \%$ confidence: 14.0-15.0 Gyr) suggests an age of about a Gyr older than the (no-backreaction) $\Lambda \mathrm{CDM}$ estimate.
\end{abstract}

Key words. cosmology: observations - cosmological parameters - distance scale - large-scale structure of Universe - dark energy

\section{Introduction}

The $\Lambda$ CDM model (the cold dark matter model with a nonzero cosmological constant $\Lambda$ ), whose metric is a member of the Friedmann-Lemaitre-Robertson-Walker (FLRW) family, is the standard cosmological model, but it assumes a non-standard model of gravity. In other words, gravity is assumed to apply separately to structure formation and FLRW uniform spatial expansion: the former is hypothesised to be gravitationally decoupled from the latter, despite the coupling present in the Raychaudhuri equation and the Hamiltonian constraint (Buchert 2000a,b, 2001). Work towards a cosmological model in which standard general relativity determines the relation between structure formation and expansion is incomplete (e.g. Ellis \& Stoeger 1987; Buchert 2011; Bolejko \& Korzyński 2017, and references therein). A common element to many implementations of this relativistic, gravitationally coupled approach to cosmology is to assume an Einstein-de Sitter model (EdS) at early times, when density perturbations are weak, and extrapolate this forward in cosmological time as a "background" model, adopting the same time foliation for an effective model that includes gravitational coupling. Here, we argue that the Hubble constant $H_{1}^{\text {bg }}$ needed to normalise this background EdS model at early epochs, such that the present effective scale factor is unity, cannot be chosen arbitrarily, since it is observationally constrained. The value of $H_{1}^{\text {bg }}$ will be needed, in particular, for $N$-body simulations that are modified to be consistent with the general-relativistic constraints imposed by scalar averaging (Roukema et al., in prep.) and for other simulational approaches working towards general-relativistic cosmology (Giblin et al. 2016a,b; Bentivegna \& Bruni 2016; Adamek et al. 2016; Rácz et al. 2016; Daverio et al. 2016; Macpherson et al. 2016).

One of the main proposals for a relativistic improvement over $\Lambda \mathrm{CDM}$ is the scalar averaging approach (Buchert 2000b, 2001, 2011), which, in general, is background-free. This approach extends the Friedmann and acceleration equations (Hamiltonian constraint and Raychaudhuri equation) from the homogeneous case to general-relativistically take into account inhomogeneous curvature and inhomogeneous expansion of the Universe (Räsänen 2004; Buchert 2008; Wiegand \& Buchert 2010; Buchert \& Räsänen 2012). This leads to a candidate explanation of dark energy being the recent emergence of average negative scalar curvature (Buchert 2005), in particular by dividing the spatial section into complementary under- and overdense regions (Buchert \& Carfora 2008; Buchert 2008; for a related phenomenological lapse function approach, see Wiltshire 2007a,b). Deviations of the average curvature from a constant-curvature model are induced by kinematical backreaction, together obeying a combined conservation law (Buchert 2000b), while implying global gravitational instability of the FLRW model and driving the average model into the dark energy sector on large scales (Roy et al. 2011).

In practice, even when developing a background-free implementation of a scalar-averaged cosmological model, an EdS 
model still provides the simplest choice for initial conditions, so that the question of choosing an observationally acceptable value of $H_{1}^{\text {bg }}$ arises. Existing implementations of emerging average negative curvature models include, among others, toy models of collapsing and expanding spheres (Räsänen 2006) or LemaîtreTolman-Bondi (LTB) regions (Nambu \& Tanimoto 2005; Kai et al. 2007), a peak model (Räsänen 2008), a metric template model (Larena et al. 2009; Chiesa et al. 2014), bi-scale or more general multi-scale models (Wiegand \& Buchert 2010; Buchert \& Räsänen 2012), the Timescape model (Wiltshire 2009; Duley et al. 2013; Nazer \& Wiltshire 2015), the virialisation approximation (Roukema et al. 2013), an effective viscous pressure approach (Barbosa et al. 2016), and Swiss cheese models that paste exact inhomogeneous solutions into holes in a homogeneous (FLRW) background (Bolejko \& Célérier 2010; the Tardis model of Lavinto et al. 2013). Updates to many of these models should benefit from an observationally justified estimate of $H_{1}^{\mathrm{bg}}$. (See also recent work on averaging of LTB Sussman et al. 2015; Chirinos Isidro et al. 2017; and Szekeres models Bolejko 2009; for evolving sign-of-curvature models, see e.g. Krasinski 1981, 1982, 1983; Stichel 2016; for averaging using Cartan scalars, see Coley 2010; Kašpar \& Svítek 2014.)

Moreover, the ratio $H_{0}^{\mathrm{bg}} / H_{0}^{\mathrm{eff}}$, where $H_{0}^{\mathrm{bg}}$ is the current value of the background EdS model Hubble parameter and $H_{0}^{\text {eff }}$ is the effective low-redshift Hubble constant (Lemaitre 1927; see also Hubble 1929), is another key property that should emerge in a relativistic cosmological model. If this ratio is as low as $H_{0}^{\text {bg }} / H_{0}^{\text {eff }} \sim 1 / 2$ (cf. Roukema et al. 2013), then, through Eqs. (7) and (8), presented below in Sect. 2.2, an observational order of unity effect on the effective density and curvature parameters is expected in comparison to their values in a decoupled (FLRW) model, so that average recent-epoch hyperbolicity (negative curvature) can provide the main component of "dark energy". This responds to the commonly raised objection to dark-energy-free scalar-averaged models, according to which the theoretically expected emerging average negative curvature is of an order of magnitude too small to explain the needed amount of dark energy, for example in the conservative approach of Buchert et al. (2013), where the overall backreaction magnitude is found to lie in the range of a few percent on large scales. (By "conservative", we mean that Buchert et al. 2013 use a generic scalar-averaging formalism, but implement it in a background-dependent way.)

Contrary to the popular conception that spatial curvature is tightly constrained observationally, observational constraints on recently emerged current average negative curvature (denoted $\Omega_{\mathcal{R} 0}^{\text {eff }}$ in Eq. (8) below) are weak. For example, Larena et al. (2009) and Sapone et al. (2014) applied the Clarkson, Bassett \& Lu test (Clarkson et al. 2008; Clarkson 2012), but found that existing catalogues are not yet accurate enough. Curvature constraints that use cosmic microwave background (CMB) and/or baryon acoustic oscillation (BAO) data and assume an FLRW model are precise in estimating the homogeneous curvature parameter $\Omega_{\mathrm{k} 0}$ to be bound by $\left|\Omega_{\mathrm{k} 0}\right| \lesssim 0.005$ (Planck Collaboration XIII 2015; or, e.g., $\left|\Omega_{\mathrm{k} 0}\right| \lesssim 0.009$, Chen et al. 2016). However, they are inaccurate in the sense that they do not allow for average comoving curvature evolution when fitting the observational data, that is, $\Omega_{\mathrm{k} 0}$ is unlikely to be a good approximation to $\Omega_{\mathcal{R} 0}^{\text {eff }}$. This restriction leads to inaccuracy because voids, which dominate the volume of the recent Universe, are generalrelativistically characterised by an average negative scalar curvature. The latter effect is mirrored by, for example, the recent growth of the virialisation fraction, which is a dimensionless parameter that can be used to measure inhomogeneity growth for the complementary overdense structures (Roukema et al. 2013).

The details of individual effective models vary. Here, we use the bi-scale scalar-averaging approach (e.g. Roukema et al. 2013, and references therein). In Sect. 2 we summarise our assumptions (Sect. 2.1) and present the key equations (Sect. 2.2). These provide relations among five present-epoch cosmological parameters and one early-epoch-normalised cosmological parameter, $H_{1}^{\mathrm{bg}}$. In Sect. 3 we use some properties of the $\Lambda \mathrm{CDM}$ model, considered as an observational proxy, to derive estimates of $H_{1}^{\mathrm{bg}}$ and $H_{0}^{\mathrm{bg}}$. Since low-redshift observational properties of the $\Lambda \mathrm{CDM}$ proxy are primarily spatial, not temporal, we discuss the consequences for the age of the Universe in Sect. 4. We quantify the challenge in estimating recently emerged curvature in Sect. 5. We conclude in Sect. 6.

\section{Implementation of scalar averaging}

We aim here to make a minimal number of assumptions. While implementations of scalar averaging and other relativistic approaches to cosmology vary, these assumptions are generally adopted, even if implicitly.

\subsection{Model assumptions}

As in several scalar-averaging implementations, such as that of Roukema et al. (2013), we assume

(i) an Einstein-de Sitter (EdS) "background" model at early times, which we extrapolate to the present; the model is parametrised by $H_{1}^{\mathrm{bg}}:=H^{\mathrm{bg}}\left(a_{\mathrm{bg}}=1\right)$, where the background scale factor $a_{\mathrm{bg}}$ and Hubble parameter $H^{\mathrm{bg}}$ are given by

$a_{\mathrm{bg}}:=\left(3 H_{1}^{\mathrm{bg}} t / 2\right)^{2 / 3}, \quad H^{\mathrm{bg}}:=\dot{a}_{\mathrm{bg}} / a_{\mathrm{bg}}=2 /(3 t)$,

and the effective scale factor $a_{\mathrm{eff}}$ (normalised to $a_{\mathrm{eff}}=1$ at the present time $\left.t_{0} \equiv t_{a_{\mathrm{eff}}=1}\right)$ satisfies $a_{\mathrm{eff}} \approx a_{\mathrm{bg}}$ at early times;

(ii) zero cosmological constant/dark energy, that is, $\Lambda:=0$;

(iii) bi-domain scalar averaging-division of a spatial slice into over- and underdense regions; and

(iv) virialisation of collapsed (overdense) regions; these are assumed to have a negligible expansion rate (stable clustering in real space, e.g. Peebles 1980; Jing 2001);

and we define

$H_{0}^{\mathrm{bg}}:=H^{\mathrm{bg}}\left(a_{\mathrm{eff}}=1\right)$.

We refer to scalar averages, denoted "eff", as "parameters", that is, for a fixed large scale of statistical homogeneity (e.g. Hogg et al. 2005; Scrimgeour et al. 2012; Wiegand et al. 2014). The EdS high-redshift assumption (i) is observationally realistic. Although $\Omega_{\mathrm{m} 0}$ is often written as $\Omega_{\mathrm{m}}$ for convenience, $\Omega_{\mathrm{m}}(z)$ in the FLRW models is (in general) $z$-dependent. In $\Lambda$ CDM, $\Omega_{\mathrm{m}}(z=1100) \approx 1-10^{-9}$ (ignoring energy density components such as radiation and neutrinos), which is observationally indistinguishable at that redshift from the EdS value of $\Omega_{\mathrm{m}}(z \approx 1100)=1$.

\subsection{Key equations}

Since the spherical collapse overdensity threshold is several hundred, volume-weighted averaging, together with assumptions 
(iii) and (iv), implies that the average expansion rate is close to that of the underdense regions, especially at late times, and can be rewritten as

$H^{\mathrm{eff}}(t) \approx H^{\mathrm{bg}}(t)+H_{\mathrm{pec}}^{\mathrm{void}}(t)$

where $H_{\text {pec }}^{\text {void }}$ is the peculiar expansion rate of voids, or in other words, the expansion rate above that of the extrapolated highredshift background EdS model (cf. Eq. (32) of Buchert \& Carfora 2008; Eq. (2.27) of Roukema et al. 2013). At early epochs, before the main virialisation epoch, the expansion is dominated by the EdS background model, that is,

$H^{\mathrm{eff}} \approx H^{\mathrm{bg}}=H_{1}^{\mathrm{bg}} a_{\mathrm{bg}}^{-3 / 2}$,

while at the present, the effective local expansion (measured by local estimates of the Hubble constant) is the sum of the background expansion rate and the peculiar expansion rate of voids, which reads

$H_{0}^{\mathrm{eff}} \approx H_{0}^{\mathrm{bg}}+H_{\mathrm{pec}, 0}^{\mathrm{void}}$.

Since we have an early-epoch EdS model that we extrapolate to later epochs (assumption (i)), matter conservation gives the effective present-day matter density parameter (e.g. Eq. (6), Buchert \& Carfora 2008)

$\Omega_{\mathrm{m} 0}^{\mathrm{eff}}=\frac{\Omega_{\mathrm{m} 0}^{\mathrm{bg}}}{\left(H_{0}^{\mathrm{eff}} / H_{0}^{\mathrm{bg}}\right)^{2}}\left(\frac{a_{\mathrm{bg} 0}}{a_{\mathrm{eff} 0}}\right)^{3}=a_{\mathrm{bg} 0}^{3}\left(\frac{H_{0}^{\mathrm{bg}}}{H_{0}^{\mathrm{eff}}}\right)^{2}$,

where $a_{\mathrm{bg} 0}, a_{\mathrm{eff} 0}$ are the current values of $a_{\mathrm{bg}}, a_{\mathrm{eff}}$, respectively. Equation (6) has solutions $H_{0}^{\mathrm{bg}}= \pm H_{0}^{\mathrm{eff}} \sqrt{\Omega_{\mathrm{m} 0}^{\mathrm{eff}} / a_{\mathrm{bg} 0}^{3}}$. A highredshift $(z \gtrsim 3)$ model that contracts would not be physically realistic, so that we have positive $H_{0}^{\mathrm{bg}}$. We have a void-dominated model, therefore we also have positive $H_{\text {pec, }, 0}^{\text {void }}$ Thus, the solution of physical interest is

$H_{0}^{\mathrm{bg}}=H_{0}^{\mathrm{eff}} \sqrt{\Omega_{\mathrm{m} 0}^{\mathrm{eff}} / a_{\mathrm{bg} 0}^{3}}$.

We can now estimate the effective scalar curvature. The Hamiltonian constraint (e.g. Eq. (7), Buchert \& Carfora 2008) at the current epoch gives

$\Omega_{\mathcal{R} 0}^{\mathrm{eff}}=1-\Omega_{\mathrm{m} 0}^{\mathrm{eff}}-\Omega_{Q 0}^{\mathrm{eff}}$,

where $\Omega_{\mathcal{R} 0}^{\mathrm{eff}}$ is the effective (averaged) present-day scalar (3Ricci) curvature parameter and $\Omega_{Q 0}^{\text {eff }}$ is the effective (averaged) present-day kinematical backreaction parameter. See for example Eq. (2.9), Roukema et al. (2013). The sum $\Omega_{\mathcal{X}}:=\Omega_{\mathcal{R}}^{\text {eff }}+\Omega_{Q}^{\text {eff }}$ (Sect. 2.4.1, Buchert 2008) is not only a relativistic alternative to dark energy on large scales, it may also provide a relativistic contribution to dark matter on small scales. As summarised in Sect. 1, observational constraints on recently evolved average spatial hyberbolicity remain weak, and we comment on this further in Sect. 5.

The FLRW equivalents of two of the parameters in Eq. (7)$H_{0}^{\mathrm{eff}}$ and $\Omega_{\mathrm{m} 0}^{\mathrm{eff}}$-have been the subject of low-redshift observational work for many decades. In Eqs. (9) and (10) below, we show that adding a third long-studied observational parameter, $t_{0}$, lets us observationally estimate both $H_{1}^{\text {bg }}$ and $H_{0}^{\text {bg }}$ when we use the $\Lambda \mathrm{CDM}$ model as a proxy, in the sense that it provides a phenomenological fit to many observations.

\section{3. ^CDM as an observational proxy}

With the aim of using $\Lambda \mathrm{CDM}$ as an extragalactic observational proxy, we can use Eq. (1) and assumption (i) to write $H_{0}^{\text {bg }}$ in alternative form to that in Eq. (7),

$H_{0}^{\mathrm{bg}}=2 /\left(3 t_{0}\right)$,

and using Eqs (1) and (7), we can now write $H_{1}^{\mathrm{bg}}$ as

$H_{1}^{\mathrm{bg}}=H_{0}^{\mathrm{eff}} \sqrt{\Omega_{\mathrm{m} 0}^{\mathrm{eff}}}$.

Thus, Eqs. (9) and (10) show that $H_{0}^{\text {bg }}$ and $H_{1}^{\text {bg }}$ are constrained by the values of $\Omega_{\mathrm{m} 0}^{\mathrm{eff}}, H_{0}^{\mathrm{eff}}$ and $t_{0}$, estimated either by methods that minimise model dependence, or by using $\Lambda \mathrm{CDM}$ as an observational proxy for these values.

Ideally, moderate- $z$ non-CMB $\Lambda C D M$ proxy estimates of $\Omega_{\mathrm{m} 0}^{\mathrm{eff}}$ and $H_{0}^{\mathrm{eff}}$ should be used in Eq. (10) in order to estimate $H_{1}^{\text {bg }}$. For example, fitting the FLRW $H(z)$ relation at moderate redshifts (e.g. $0.1 \lesssim z \lesssim 10$ ) determined by differential oldest-passive-galaxy stellar-population age dating ("cosmic chronometers"; Jimenez \& Loeb 2002) and using the FLRW fitted values of $\Omega_{\mathrm{m} 0}^{\mathrm{eff}}$ and $H_{0}^{\text {eff }}$ would be an observational strategy with only weak FLRW model dependence, especially if the technique became viable with $z \gtrsim 3$ galaxies. For galaxies with $z<3$, some authors find no significant inconsistency with $\Lambda C D M($ e.g. Moresco et al. 2016), while others find tentative evidence for a non- $\Lambda$ CDM $H(z)$ relation (Ding et al. 2015; see also the BAO estimates of Sahni et al. 2014). Here, our main aim is to illustrate our method, so for simplicity, we adopt $\Lambda \mathrm{CDM}$ as a proxy for a wide (although not complete, e.g. Bull et al. 2016; Buchert et al. 2016) range of extragalactic observations. This should provide a reasonable initial estimate of $H_{1}^{\mathrm{bg}}$. Adopting Planck values of $\Omega_{\mathrm{m} 0}=0.309 \pm 0.006, H_{0}=67.74 \pm 0.46 \mathrm{~km} \mathrm{~s}^{-1} / \mathrm{Mpc}$ (Table 4, sixth data column, Planck Collaboration XIII 2015), Eq. (10) gives

$H_{1}^{\mathrm{bg}}=37.7 \pm 0.4 \mathrm{~km} \mathrm{~s}^{-1} / \mathrm{Mpc}$,

where, for the sake of illustration, the errors in the $\Omega_{\mathrm{m} 0}$ and $H_{0}$ estimates are assumed to be Gaussian and independent, with zero covariance. (The recent discovery of differential space expansion on the hundred-megaparsec scale around our Galaxy Bolejko et al. 2016; see also Kraljic \& Sarkar 2016, and the percentlevel deviation of the Riess et al. 2016, estimate of $H_{0}^{\text {eff }}$ from the Planck estimate indicate percent-level effects on $H_{0}^{\text {eff }}$ when averaging on the hundred-megaparsec scale (see also Ben-Dayan et al. 2014), which would modify the estimates presented here at a similarly weak level.) After submission of this paper, we found that Rácz et al. (2016) derived an almost identical $\Lambda$ CDM-proxy value of $H_{1}^{\text {bg }}$.

Similarly, Eq. (9), using the Planck age of the Universe estimate $t_{0}^{\Lambda \mathrm{CDM}}=13.80 \pm 0.02 \mathrm{Gyr}$ as a proxy, yields

$H_{0}^{\mathrm{bg}}=47.24 \pm 0.07 \mathrm{~km} \mathrm{~s}^{-1} / \mathrm{Mpc}$.

This is significantly higher than direct EdS fits to the CMB with broken-power-law or bump primordial spectra (Blanchard et al. 2003; Hunt \& Sarkar 2007; Nadathur \& Sarkar 2011), for example $43.3 \pm 0.9 \mathrm{~km} \mathrm{~s}^{-1} / \mathrm{Mpc}$ for what in our terminology appears to correspond to $H_{0}^{\text {bg }}$ (Hunt \& Sarkar 2010, Table 2). 
Comparison of Eqs. (7) and (10) gives the corresponding present-day background scale factor

$a_{\mathrm{bg} 0}=\left(H_{1}^{\mathrm{bg}} / H_{0}^{\mathrm{bg}}\right)^{2 / 3}=0.860 \pm 0.007$,

which is slightly stronger than the $\approx 10 \%$ shrinkage in the BAO peak location detected for Sloan Digital Sky Survey luminous red galaxy pairs whose paths cross superclusters in either the Nadathur \& Hotchkiss (2014) or Liivamägi et al. (2012) supercluster catalogues (Roukema et al. 2015, 2016). This suggests that BAO-peak-scale regions crossing superclusters could be considered as a slightly expanded present-day physical realisation of the EdS model extrapolated from early epochs, which we refer to in this paper as our background model. The values discussed below in Sect. 4 yield $a_{\mathrm{bg} 0}=0.90 \pm 0.01$, in which case the EdS background model and the BAO-peak-scale regions crossing superclusters correspond even more closely.

\section{Astrophysical age of universe estimates as a test of inhomogeneous cosmology}

The value of $H_{1}^{\text {bg }}$ in Eq. (11) gives $t_{a_{\mathrm{bg}}=1}=17.3 \mathrm{Gyr}$ (cf. Eq. (1)). In a scalar averaging model, $a_{\mathrm{eff}}(t)>a_{\mathrm{bg}}(t)$ (and $H^{\text {eff }}(t)>$ $\left.H^{\mathrm{bg}}(t)\right)$ are expected, especially during the structure formation epoch, so the expected current age of the Universe should be lower, that is, $t_{0}<17.3 \mathrm{Gyr}$. A model that provides $a_{\mathrm{eff}}=1$ at 13.8 Gyr would closely match $\Lambda$ CDM. However, by evolving an initial power spectrum of density perturbations from an early epoch forward in foliation time, predictions of $t_{0}$ that differ from the $\Lambda \mathrm{CDM}$ value can also be made. For example, this evolution can be calculated using the relativistic Zel'dovich approximation (Kasai 1995; Morita et al. 1998) in the form given by Buchert \& Ostermann (2012), Buchert et al. (2013), Alles et al. (2015); see also Matarrese \& Terranova (1996), Villa et al. (2011).

Use of $\Lambda$ CDM as a proxy in Sect. 3 can be considered to be approximately calibrated by differential passive galaxy age dating, which relates the effective scale factor and the time foliation, at redshifts $0.1 \lesssim z \lesssim 2$. However, at low redshifts, observational constraints on $\Lambda \mathrm{CDM}$ mostly do not directly relate to the time foliation. For example, "observed peculiar velocities" are combinations of spectroscopic redshifts, distance estimators, and an assumed value of $H_{0}$; they are not measured spatial displacements differentiated with respect to measured foliation time. Moreover, Ly $\alpha$ BAO estimates for $H^{\text {eff }}(z \sim 2.34)$ in the radial direction are about $7 \%$ lower than the $\Lambda \mathrm{CDM}$ expected value (Delubac et al. 2015), suggesting an underestimate of similar magnitude when using $\Lambda \mathrm{CDM}$ as a proxy to estimate $t_{0}$. In other words, it is premature to claim that $t_{0}$ is accurately estimated to within $\pm 0.1 \mathrm{Gyr}$ by $t_{0}^{\Lambda \mathrm{CDM}}=13.8 \mathrm{Gyr}$.

How well is $t_{0}$ observationally constrained? Here, we consider the integral of proper time on our Galaxy's world line from the initial singularity to the present to be negligibly different from the corresponding time interval in terms of coordinate time $t$, so that both can be consistently denoted by $t_{0}$ (see, however, Nazer \& Wiltshire 2015, and references therein). Six microlensed bulge $\sim 1 M_{\odot}$ stars have most-probable age estimates in the range 14.2-14.7 Gyr (Table 5, Bensby et al. 2013). The probability density functions (pdfs) of the age estimates for these stars are highly asymmetric, with $68 \%$ confidence intervals each including $t_{0}^{\Lambda \mathrm{CDM}}$ and having an upper bound below or nearly below the mode of the pdf.

A more precise estimate of a lower bound to $t_{0}$ can be obtained if we interpret the pdfs of these microlensed stars to represent the current best state of our knowledge of the ages of these

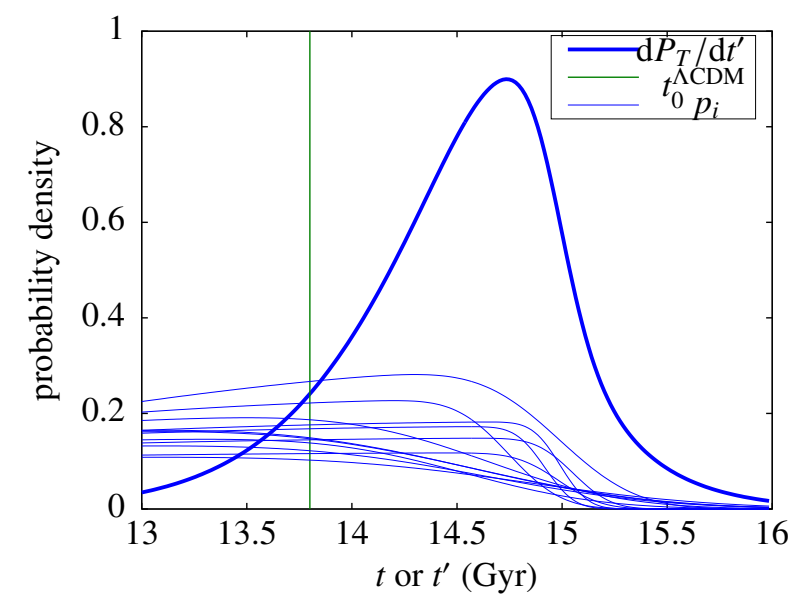

Fig. 1. Skew-normal reconstructed (Eq. (14)) probability density functions $p_{i}$ of the 12 Bensby et al. (2013) microlensed Galactic bulge stars whose most likely age is greater than $13.0 \mathrm{Gyr}$ (thin curves); and probability density function $\mathrm{d} P_{T} / \mathrm{d} t^{\prime}$ of the most likely oldest age $T$ of these stars (thick curve), assuming that the true stellar ages are chosen randomly from their respective pdfs $p_{i}$ (Eq. (15)). The vertical line indicates $t_{0}^{\Lambda \mathrm{CDM}}$.

stars. In that case, we can remodel the published distributions using skew-normal distributions, that is, with pdfs

$p(t ; \alpha, \xi, \omega)=\frac{\mathrm{e}^{-\tau^{2} / 2}}{\omega \sqrt{2 \pi}}\left[1+\operatorname{erf}\left(\frac{\alpha \tau}{\sqrt{2}}\right)\right]$,

where $\tau:=(t-\xi) / \omega$ is a rescaled age, $\xi$ is a location parameter, $\omega$ is a width parameter, $\alpha$ is an asymmetry parameter (de Helguero 1909; Birnbaum 1950; Azzalini 2005). We numerically search for (using simulated annealing) the triple $(\alpha, \xi, \omega)$ that best matches the age pdf parameters in Cols. 5-7 of Table 5 of Bensby et al. (2013) for each of the stars whose most likely age (Col. 5) is greater than $13.0 \mathrm{Gyr}$, yielding $p_{i}$ for star $i=1, \ldots, 12$ satisfying this definition of old stars. The cumulative probability $P_{T}$ that none of the 12 stars is older than an age $t^{\prime}$ is

$P_{T}\left(T \leq t^{\prime}\right)=\Pi_{i=1,12} \int_{-\infty}^{t^{\prime}} p_{i}(t) \mathrm{d} t$.

As shown in Fig. 1, this gives the most likely value for $T$, the age of the oldest star in this sample, as $T=14.7_{-0.7}^{+0.3} \mathrm{Gyr}$ (the uncertainties indicate the $68 \%$ central confidence interval in $P_{T}$; or $14.7_{-1.5}^{+0.8} \mathrm{Gyr}$, at $95 \%$ ). This value provides a lower bound for $t_{0}$; assuming this oldest star formed very early in the Universe, that is, $T \approx t_{0}$, would give $H_{0}^{\mathrm{bg}}=44_{-2}^{+1} \mathrm{~km} \mathrm{~s}^{-1} / \mathrm{Mpc}$, which is about $3 \mathrm{~km} \mathrm{~s}^{-1} / \mathrm{Mpc}$ lower than the value in Eq. (12) and consistent with the CMB estimate of Hunt \& Sarkar (2010) of $43.3 \pm 0.9 \mathrm{~km} \mathrm{~s}^{-1} / \mathrm{Mpc}$ quoted above. The latter type of estimate appears to be convergent with respect to increasing data quality and improved analysis (Blanchard et al. 2003; Hunt \& Sarkar 2007; Nadathur \& Sarkar 2011). As mentioned above, this lower estimate of $H_{0}^{\mathrm{bg}}$ yields $a_{\mathrm{bg} 0}=0.90 \pm 0.01$, improving the correspondence between the BAO peak shift (shrinkage across superclusters; Roukema et al. 2015, 2016) and $a_{\mathrm{bg} 0}$.

With improved stellar modelling accuracy, estimates of this sort can potentially be used to distinguish $\Lambda \mathrm{CDM}$ from relativistic inhomogeneous models. However, the present derivation of $P_{T}$ by analysing the Bensby et al. (2013) microlensed stars is not sufficient to reject $\Lambda$ CDM. Firstly, the probability of having 
$T \leq t_{0}^{\Lambda \mathrm{CDM}}$ according to $P_{T}$ is $9 \%$, which is not a statistically significant rejection. Secondly, and more importantly, $P_{T}$ as defined here is designed to provide the pdf of a best estimate of a lower bound to $t_{0}$, given the published modelling of the observational data and assuming that the individual stellar pdfs are statistically independent; it is not designed to test the hypothesis that $t_{0}^{\Lambda \mathrm{CDM}}$ is the true value of $t_{0}$. Thirdly, the typical uncertainties in stellar age modelling represented in the pdfs $p_{i}$ are high. For example, Fig. 12 of Dotter et al. (2007) shows variation in age of up to about $\pm 15 \%$ if oxygen and iron abundances are $0.3 \mathrm{dex}$ higher than what the authors refer to as "scaled-solar" abundances; and Figs. 15 and 16 of VandenBerg et al. (2012) indicate that a 0.4 dex enhancement in magnesium or silicon abundance has a stronger effect on effective temperature $T_{\text {eff }}$ than 2 Gyr in age. In particular, VandenBerg et al. (2014) estimate the age of the halo subgiant HD 140283 as $14.3 \pm 0.4 \mathrm{Gyr}$, where the error is predominantly a parallax error, or $14.3 \pm 0.8 \mathrm{Gyr}$, including all sources of uncertainty, such as that of the oxygen abundance.

\section{Present-day/recent average curvature}

As stated above, the present-day large-scale mean curvature represented in Eq. (8) is difficult to measure (e.g., Larena et al. 2009; Sapone et al. 2014; Räsänen et al. 2015), but will become measurable in near-future surveys such as Euclid (Refregier et al. 2010), DESI (Dark Energy Spectroscopic Instrument; Levi et al. 2013), 4MOST (4-metre Multi-Object Spectroscopic Telescope; de Jong et al. 2012, 2014), eBOSS (extended Baryon Oscillation Spectroscopic Survey; Zhao et al. 2016), LSST (Large Synoptic Survey Telescope; Tyson et al. 2003), and HETDEX (Hobby-Eberly Telescope Dark Energy eXperiment; Hill et al. 2008). Power-law models of dark-energyfree (cf. (ii)) backreaction evolution tend to give a strong negative mean curvature (positive $\Omega_{\mathcal{R} 0}^{\text {eff }}$ ), for example, Wiegand \& Buchert (Sect. IV, 2010) argue for $\Omega_{\mathcal{R} 0}^{\text {eff }} \approx 1.05, \Omega_{Q 0}^{\text {eff }} \approx-0.35$. For the purposes of illustration, we set $\Omega_{Q 0}^{\text {eff }}=0$ in this paragraph, giving a slightly weaker negative mean present-day curvature, with $\Omega_{\mathcal{R} 0}^{\text {eff }} \approx 0.7$ from Eq. (8). If a volume with $\Omega_{\mathcal{R} 0}^{\text {eff }} \approx 0.7$ is represented as a single time-slice constant-curvature ("template") model (Buchert \& Carfora 2002; or alternatively, by smoothing the curvature and neglecting the "dressing" of Riemannian volume and the subdominant curvature fluctuation backreaction that both arise through the smoothing process; see Buchert \& Carfora 2003 ), then we can write an effective curvature radius $R_{\mathrm{C} 0}^{\mathrm{eff}} \approx$ $\left(c / H_{0}^{\mathrm{eff}}\right) \Omega_{\mathcal{R} 0}^{\mathrm{eff}}-1 / 2 \approx 3580 \mathrm{~h}^{-1} \mathrm{Mpc}$. Still assuming constant curvature, the difference between the expected tangential arclength subtending a radian $r_{\perp}^{-}$and a flat space arclength $r_{\perp}^{0}$ at a small radial comoving distance $r$ is $r_{\perp}^{-}-r_{\perp}^{0}=R_{\mathrm{C} 0}^{\text {eff }} \sinh \left(r / R_{\mathrm{C} 0}^{\text {eff }}\right)-r \approx$ $(1 / 6) r\left(r / R_{\mathrm{C} 0}^{\mathrm{eff}}\right)^{2}$ to highest order. Thus, up to $300 h^{-1} \mathrm{Mpc}$ from the observer, the tangential "stretch" is less than about $0.1 \%$, that is, BAO curvature constraints would have to be accurate to better than $\pm 0.1 h^{-1} \mathrm{Mpc}$. At a redshift $z=1$, the tangential effect should be stronger, but in the past light cone, the present-day averaged curvature does not apply. Instead, using $\Lambda$ CDM as a proxy, we should have $\Omega_{\mathcal{R}}^{\text {eff }}(z=1) \approx \Omega_{\Lambda}(z=1)=0.23$, that is, the average negative curvature is weaker, with a constantcurvature curvature radius. (We use the adjective "constantcurvature" in front of "curvature radius" to emphasise that interpreting the mean spatial curvature in terms of a curvature radius is only meaningful for some types of approximate calculations, such as for small angles.) $R_{\mathrm{C}}^{\text {eff }}(z=1) \approx 6310 h^{-1} \mathrm{Mpc}$, double the local value. In a constant cosmological time, constant-curvature hypersurface at $z=1$, the amount of tangential stretching that should occur at $500 \mathrm{~h}^{-1} \mathrm{Mpc}$ from the observer is thus about $0.1 \%$. At the BAO scale of about $105 h^{-1} \mathrm{Mpc}$, stretching would be about 25 times weaker. It remains to be determined whether $\sim 0.004 \%$ stretching will be detectable in the coming decade of major observational projects such as Euclid, DESI, 4MOST, eBOSS, LSST, and HETDEX.

\section{Conclusion}

Equations (1)-(10) provide a summary of the key relationships between present-day observational parameters of the bidomain scalar averaging model, satisfying assumptions (i)-(iv). The peculiar expansion rate of voids was previously estimated as $H_{\mathrm{pec}, 0}^{\mathrm{void}} \approx 36 \pm 3 \mathrm{~km} \mathrm{~s}^{-1} / \mathrm{Mpc}$ (Eq. (2.36), Roukema et al. 2013) from corresponding surveys of galaxy clusters and voids, but Eqs. (5) and (12) imply that this is an overestimate by $\sim 10 \mathrm{~km} \mathrm{~s}^{-1} / \mathrm{Mpc}$, even taking into account a somewhat greater $t_{0}$ motivated by stellar estimates. Thus, following a scalar averaging (or similar) approach, $H_{\text {pec }, 0}^{\text {void }}$, together with the early-epochnormalised background EdS Hubble constant $H_{1}^{\text {bg }}$, the current background Hubble parameter $H_{0}^{\text {bg }}$, the effective Hubble constant $H_{0}^{\text {eff }}$, the effective matter density parameter $\Omega_{\mathrm{m} 0}^{\mathrm{eff}}$, and the age of the Universe $t_{0}$ form a closely linked sextuple. Estimates of $H_{0}^{\text {eff }}$ and $\Omega_{\mathrm{m} 0}^{\mathrm{eff}}$ are generally uncontroversial, though usually interpreted in terms of the gravitationally decoupled (standard) cosmological model.

Here, we have shown that since $H_{1}^{\text {bg }}$ is physically realised at high redshift rather than low redshift, it can be given a value with a reasonable observational justification, using $\Lambda \mathrm{CDM}$ as an observational proxy. The Planck $\Lambda \mathrm{CDM}$ values yield $H_{1}^{\mathrm{bg}}=$ $37.7 \pm 0.4 \mathrm{~km} \mathrm{~s}^{-1} / \mathrm{Mpc}$ (Eq. (11)). The corresponding recently emerged average negative spatial scalar curvature, represented by the effective curvature parameter $\Omega_{\mathcal{R} 0}^{\mathrm{eff}}$, is currently not constrained by observations, and constitutes a key challenge for observational cosmology in the coming decades.

The corresponding high value of $t_{a_{\mathrm{bg}}=1}=17.3 \mathrm{Gyr}$ motivates refocussing attention on astrophysical age estimates such as the microlensed oldest bulge star estimate $T=14.7_{-0.7}^{+0.3} \mathrm{Gyr}$ discussed above, since standard cosmological tools do not seem to provide CMB-free estimates of $t_{0}$. Modelling of suspectedoldest stars with an appropriate statistical approach and observational strategies could potentially result in a stellar rejection of $\Lambda$ CDM (cf. VandenBerg et al. 2014). As cosmological models with standard (Einstein) gravity continue to be refined, predictions of $t_{0}$ will need to be made and compared to improved stellar constraints.

Since the order of magnitude of cosmological backreaction effects is often claimed to be tiny, the following order-ofmagnitude summary of Eqs. (4)-(8) and (10) and their values may help underline the inaccuracy implied by ignoring standard Einsteinian gravity:

$\frac{2}{3} \approx \frac{H_{0}^{\mathrm{bg}}}{H_{0}^{\mathrm{eff}}} \approx \frac{H_{1}^{\mathrm{bg}}}{H_{0}^{\mathrm{eff}}} \approx \sqrt{\Omega_{\mathrm{m} 0}^{\mathrm{eff}}}=\sqrt{1-\Omega_{\mathcal{R} 0}^{\mathrm{eff}}-\Omega_{Q 0}^{\mathrm{eff}}} \approx \frac{1}{2} \gtrsim \frac{H_{\mathrm{pec}, 0}^{\mathrm{void}}}{H_{0}^{\mathrm{eff}}}$,

for example, it is observationally realistic for the effective expansion rate to be as much as twice the background expansion rate. Accepting $H_{0}^{\text {eff }}$ and $\Omega_{\mathrm{m} 0}^{\text {eff }}$ as approximately well-known, observational estimation of $H_{0}^{\mathrm{bg}}$ only depends on estimating $t_{0}$; $H_{1}^{\text {bg }}$ is the main theme of this paper; $\Omega_{\mathcal{R} 0}^{\text {eff }}$ is only weakly constrained, although a strong positive value is expected due to 
the spatial dominance of voids; $\Omega_{Q 0}^{\mathrm{eff}}$ is, in principle, measurable from distance-redshift catalogues; and $H_{\text {pec, } 0}^{\text {void }}$ was estimated in Roukema et al. (2013) but remains open to improved methods.

Acknowledgements. Thank you to Roland Bacon, Laurence Tresse, Ariane Lançon, Johan Richard, Subir Sarkar, David Wiltshire, Thomas Bensby and an anonymous referee for useful comments. The work of T.B. and P.M. was conducted within the "Lyon Institute of Origins" under grant ANR-10-LABX-66. A part of this project was funded by the National Science Centre, Poland, under grant 2014/13/B/ST9/00845. J.J.O. benefited from doctoral stipendium financial support under grant DEC-2014/12/T/ST9/00100 of the National Science Centre, Poland. Part of this work consists of research conducted within the scope of the HECOLS International Associated Laboratory, supported in part by the Polish NCN grant DEC-2013/08/M/ST9/00664. A part of this project has made use of computations made under grant 197 of the Poznań Supercomputing and Networking Center (PSNC).

\section{References}

Adamek, J., Daverio, D., Durrer, R., \& Kunz, M. 2016, Nat. Phys., 12, 346 Alles, A., Buchert, T., Al Roumi, F., \& Wiegand, A. 2015, Phys. Rev. D, 92 023512

Azzalini, A. 2005, Scand. J. Stat., 32, 159

Barbosa, R. M., Chirinos Isidro, E. G., Zimdahl, W., \& Piattella, O. F. 2016, Gen. Relat. Grav., 48, 51

Ben-Dayan, I., Durrer, R., Marozzi, G., \& Schwarz, D. J. 2014, Phys. Rev. Lett. 112,221301

Bensby, T., Yee, J. C., Feltzing, S., et al. 2013, A\&A, 549, A147

Bentivegna, E., \& Bruni, M. 2016, Phys. Rev. Lett., 116, 251302

Birnbaum, Z. W. 1950, Ann. Math. Stat., 21, 272

Blanchard, A., Douspis, M., Rowan-Robinson, M., \& Sarkar, S. 2003, A\&A, 412, 35

Bolejko, K. 2009, J. Cosmol. Astropart. Phys., 41, 1585

Bolejko, K., \& Célérier, M.-N. 2010, Phys. Rev. D, 82, 103510

Bolejko, K., \& Korzyński, M. 2017, ArXiv e-prints [arXiv: 1612 . 08222]

Bolejko, K., Nazer, M. A., \& Wiltshire, D. L. 2016, J. Cosmol. Astropart., 06 035

Buchert, T. 2000a, in Proc. Nineth workshop on general relativity and gravitation, Hiroshima Nov. 3-6, 1999, eds. Y. Eriguchi, T. Futamase, A. Hosoya et al. (Hiroshima: Physics Dept., Hiroshima University), 306

Buchert, T. 2000b, Gen. Rel. Grav., 32, 105

Buchert, T. 2001, Gen. Rel. Grav., 33, 1381

Buchert, T. 2005, Class. Quant. Grav., 22, L113

Buchert, T. 2008, Gen. Rel. Grav., 40, 467

Buchert, T. 2011, Class. Quant. Grav., 28, 164007

Buchert, T., \& Carfora, M. 2002, Class. Quant. Grav., 19, 6109

Buchert, T., \& Carfora, M. 2003, Phys. Rev. Lett., 90, 031101

Buchert, T., \& Carfora, M. 2008, Class. Quant. Grav., 25, 195001

Buchert, T., \& Ostermann, M. 2012, Phys. Rev. D, 86, 023520

Buchert, T., \& Räsänen, S. 2012, Ann. Rev. Nucl. Part. Sci., 62, 57

Buchert, T., Nayet, C., \& Wiegand, A. 2013, Phys. Rev. D, 87, 123503

Buchert, T., Coley, A. A., Kleinert, H., Roukema, B. F., \& Wiltshire, D. L. 2016, Int. J. Mod. Phys. D, 25, 1630007

Bull, P., Akrami, Y., Adamek, J., et al. 2016, Physics of the Dark Universe, 12, 56

Chen, Y., Ratra, B., Biesiada, M., Li, S., \& Zhu, Z.-H. 2016, ApJ, 829, 61

Chiesa, M., Maino, D., \& Majerotto, E. 2014, J. Cosmol. Astropart., 12, 49

Chirinos Isidro, E. G., Barbosa, R. M., Piattella, O. F., \& Zimdahl, W. 2017, Class. Quant. Grav., 34, 035001

Clarkson, C. 2012, Comptes Rendus Physique, 13, 682

Clarkson, C., Bassett, B., \& Lu, T. H.-C. 2008, Phys. Rev. Lett., 101, 011301

Coley, A. A. 2010, Class. Quant. Grav., 27, 245017

Daverio, D., Dirian, Y., \& Mitsou, E. 2016, ArXiv e-prints [arXiv: 1611.03437]

de Helguero, F. 1909, in Atti del IV Congresso Internazionale dei Matematici, Vol. III (sez. III-b), eds. G. Castelnuovo (Rome: R. Accademia dei Lincei)

de Jong, R. S., Bellido-Tirado, O., Chiappini, C., et al. 2012, in Ground-based and Airborne Instrumentation for Astronomy IV, eds. I. S. McLean, S. K. Ramsay, \& H. Takami, SPIE Conf. Ser. , 8446, 84460T

de Jong, R. S., Barden, S., Bellido-Tirado, O., et al. 2014, in Ground-based and Airborne Instrumentation for Astronomy V, eds. S. K. Ramsay, I. S. McLean, \& H. Takami, SPIE Conf. Ser., 9147, 91470

Delubac, T., Bautista, J. E., Busca, N. G., et al. 2015, A\&A, 574, A59

Ding, X., Biesiada, M., Cao, S., Li, Z., \& Zhu, Z.-H. 2015, ApJ, 803, L22

Dotter, A., Chaboyer, B., Ferguson, J. W., et al. 2007, ApJ, 666, 403

Duley, J. A. G., Nazer, M. A., \& Wiltshire, D. L. 2013, Class. Quant. Grav., 30, 175006
Ellis, G. F. R., \& Stoeger, W. 1987, Class. Quant. Grav., 4, 1697

Giblin, Jr, J. T., Mertens, J. B., \& Starkman, G. D. 2016a, Phys. Rev. Lett., 116, 251301

Giblin, Jr, J. T., Mertens, J. B., \& Starkman, G. D. 2016b, ApJ, 833, 247

Hill, G. J., Gebhardt, K., Komatsu, E., et al. 2008, Panoramic Views of Galaxy Formation and Evolution, eds. T. Kodama, T. Yamada, \& K. Aoki, ASP Conf. Ser., 399, 115

Hogg, D. W., Eisenstein, D. J., Blanton, M. R., et al. 2005, ApJ, 624, 54

Hubble, E. 1929, Proc. Nat. Acad. Sci., 15, 168

Hunt, P., \& Sarkar, S. 2007, Phys. Rev. D, 76, 123504

Hunt, P., \& Sarkar, S. 2010, MNRAS, 401, 547

Jimenez, R., \& Loeb, A. 2002, ApJ, 573, 37

Jing, Y. P. 2001, ApJ, 550, L125

Kai, T., Kozaki, H., Nakao, K., Nambu, Y., \& Yoo, C. 2007, Prog. Theor. Phys., 117,229

Kasai, M. 1995, Phys. Rev. D, 52, 5605

Kašpar, P., \& Svítek, O. 2014, Class. Quant. Grav., 31, 095012

Kraljic, D., \& Sarkar, S. 2016, JCAP, 10, 016

Krasinski, A. 1981, Gen. Relativ. Grav., 13, 1021

Krasinski, A. 1982, in The Birth of the Universe, eds. J. Audouze, \& J. Tran Thanh van, 15

Krasinski, A. 1983, Gen. Relativ. Grav., 15, 673

Larena, J., Alimi, J.-M., Buchert, T., Kunz, M., \& Corasaniti, P.-S. 2009, Phys. Rev. D, 79, 083011

Lavinto, M., Räsänen, S., \& Szybka, S. J. 2013, J. Cosmol. Astropart. Phys., 12, 51

Lemaître, G. 1927, Ann. Soc. Sc. Brux., 47, 49

Levi, M., Bebek, C., Beers, T., et al. 2013, ArXiv e-prints [arXiv: 1308. 0847]

Liivamägi, L. J., Tempel, E., \& Saar, E. 2012, A\&A, 539, A80

Macpherson, H. J., Lasky, P. D., \& Price, D. J. 2016, Phys. Rev. D, submitted [arXiv: 1611.05447]

Matarrese, S., \& Terranova, D. 1996, MNRAS, 283, 400

Moresco, M., Jimenez, R., Verde, L., et al. 2016, JCAP, 12, 039

Morita, M., Nakamura, K., \& Kasai, M. 1998, Phys. Rev. D, 57, 6094

Nadathur, S., \& Hotchkiss, S. 2014, MNRAS, 440, 1248

Nadathur, S., \& Sarkar, S. 2011, Phys. Rev. D, 83, 063506

Nambu, Y., \& Tanimoto, M. 2005, Gen. Relat. Quant. Cosmol., [arXiv:gr-qc/0507057]

Nazer, M. A., \& Wiltshire, D. L. 2015, Phys. Rev. D, 91,063519

Peebles, P. J. E. 1980, Large-Scale Structure of the Universe (Princeton University Press)

Planck Collaboration XIII. 2016, A\&A, 594, A13

Rácz, G., Dobos, L., Beck, R., Szapudi, I., \& Csabai, I. 2016, ArXiv e-prints [arXiv: 1607.08797]

Räsänen, S. 2004, J. Cosmol. Astropart. Phys., 2, 003

Räsänen, S. 2006, J. Cosmol. Astropart. Phys., 11, 003

Räsänen, S. 2008, J. Cosmol. Astropart. Phys., 4, 026

Räsänen, S., Bolejko, K., \& Finoguenov, A. 2015, Phys. Rev. Lett., 115, 101301

Refregier, A., Amara, A., Kitching, T. D., et al. 2010, ArXiv e-prints [arXiv: 1001.0061$]$

Riess, A. G., Macri, L. M., Hoffmann, S. L., et al. 2016, ApJ, 826, 56

Roukema, B. F., Ostrowski, J. J., \& Buchert, T. 2013, J. Cosmol. Astropart. Phys., 10,043

Roukema, B. F., Buchert, T., Ostrowski, J. J., \& France, M. J. 2015, MNRAS, 448, 1660

Roukema, B. F., Buchert, T., Fujii, H., \& Ostrowski, J. J. 2016, MNRAS, 456, L45

Roy, X., Buchert, T., Carloni, S., \& Obadia, N. 2011, Class. Quant. Grav., 28, 165004

Sahni, V., Shafieloo, A., \& Starobinsky, A. A. 2014, ApJ, 793, L40

Sapone, D., Majerotto, E., \& Nesseris, S. 2014, Phys. Rev. D, 90, 023012

Scrimgeour, M. I., Davis, T., Blake, C., et al. 2012, MNRAS, 425, 116

Stichel, P. C. 2016, ArXiv e-prints [arXiv: 1601.07030]

Sussman, R. A., Hidalgo, J. C., Dunsby, P. K. S., \& German, G. 2015, Phys. Rev. D, 91, 063512

Tyson, J. A., Wittman, D. M., Hennawi, J. F., \& Spergel, D. N. 2003, Nucl. Phys. B Proc. Supp., 124, 21

VandenBerg, D. A., Bergbusch, P. A., Dotter, A., et al. 2012, ApJ, 755, 15 VandenBerg, D. A., Bond, H. E., Nelan, E. P., et al. 2014, ApJ, 792, 110 Villa, E., Matarrese, S., \& Maino, D. 2011, J. Cosmol. Astropart. Phys., 8, 024 Wiegand, A., \& Buchert, T. 2010, Phys. Rev. D, 82, 023523

Wiegand, A., Buchert, T., \& Ostermann, M. 2014, MNRAS, 443, 241

Wiltshire, D. L. 2007a, New J. Phys., 9, 377

Wiltshire, D. L. 2007b, Phys. Rev. Lett., 99, 251101

Wiltshire, D. L. 2009, Phys. Rev. D, 80, 123512

Zhao, G.-B., Wang, Y., Ross, A. J., et al. 2016, MNRAS, 457, 2377 